\title{
Zenithal dependence of muon intensity
}

\author{
M. Nunes*, E. Kemp, B. Daniel, L. M. Santos, T. V. Vieira \\ UNICAMP - State University of Campinas - Brazil \\ E-mail: mosnuneseifi.unicamp.br
}

The zenital dependence of muon intensity which reaches the earth's surface is well known as proportional to $\cos ^{n}(\theta)$. Generally, for practical purposes and simplicity in calculations, $\mathrm{n}$ is taken as 2. However, compilations of measurements show dependence on the geographical location of the experiments as well as the muons energy range. Since analytical solutions appear to be increasingly less necessary because of the higher accessibility to low cost computational power, accurate and precise determination of the value of the exponent $n$, under different conditions, can be useful in the necessary calculations to estimate signals and backgrounds, either for terrestrial and underground experiments. In this work we discuss a method for measuring $\mathrm{n}$ using a simple muon telescope and the results obtained for measurements taken at Campinas (SP), Brazil (22 $54^{\prime} \mathrm{W},-41^{\circ} 03^{\prime}, 854 \mathrm{~m}$ asl) and at Fermilab - Batavia (IL), United States (41.83, 88.26Ł, $220 \mathrm{~m}$ asl). After validation of the method, we intend to extend the measurements for more geographic locations due to the simplicity of the method, and thus collect more values of $n$ that currently exist in compilations of general data on cosmic rays.

The 34th International Cosmic Ray Conference,

30 July- 6 August, 2015

The Hague, The Netherlands

${ }^{*}$ Speaker. 


\section{Introduction}

Muons represent $10 \%$ of the energy of an extensive air shower and because of their heavy mass and relativistic behavior, are the most abundant particles at ground level produced by the cosmic radiation. Therefore, they allow the indirect detection of cosmic rays in the surface, trough the reconstruction of the air shower, using data took by different detectors working in time coincidence to infer the direction of the primary particle. This technique was successfully used in many experiments, like the Pierre Auger Observatory [1] for ultra-high energies. Muons also represent a source of noise in other particle physics experiments, like underground neutrino detectors. For these reasons it is very important to study their flux at the surface.

The muon intensity has a peculiarity: it describes an anisotropic angular distribution that goes with a $\cos ^{n} \theta, \theta$ being the zenith angle. This feature was widely discussed, especially the parameter $n$ which was established [2] as 2. But there is no reason for this assumption to be generalized. This value for the parameter may depend on the region of the planet, altitude and period of the measurements, and also be related to the energy range considered.

This work proposes a simple experimental method to measure $n$, as a first step to study the muon intensity in details and check the dependences aforementioned. It makes use of a muon telescope made of three paddle shaped scintillator detectors, vertically aligned, operating in time coincidence. Counting rate measurements are made for different distances between the outermost paddles. Each distance defines an aperture for the telescope, which is related to the geometry of the telescope and the zenith dependence of the intensity of incoming particles.

In next session, a theoretical approach to the telescope aperture $\Gamma$ and the muon intensity are set forth. Section 3 shows the method developed to obtain $n$. Finally, section 4 describes the experimental setup used and the results obtained.

\section{Aperture of a particle telescope}

A vertical arrangement of detectors, at a fixed distance between them, defines an acceptable range of zenital angles that grants the collection of particles striking the detectors. This is given by the aperture ${ }^{1}$ of the telescope, $\Gamma$. To define it we must write an expression for the counting rate of the telescope [3]:

$$
\begin{array}{r}
C\left(\vec{x}, t_{0}\right)=\int_{t_{0}}^{t_{0}+T} d t \int_{S} d \vec{\sigma} \cdot \hat{r} \int_{\Omega} d \omega \int_{0}^{\infty} d E \times \\
\quad \times \sum_{\alpha} \varepsilon_{\alpha}(E, \vec{\sigma}, \omega, t) \vec{J}_{\alpha}(E, \omega, \vec{x}, t)
\end{array}
$$

where

- $C$ is the counting rate in $s^{-1}$ during a time interval $t$ between $t_{0}$ and $t_{0}+T$,

- $J_{\alpha}$ and $\varepsilon_{\alpha}$ are, respectively, the spectral intensity and the detection efficiency of the $\alpha^{\text {th }}$ particle in $s^{-1} \mathrm{~cm}^{-2} s r^{-1} E^{-1}$,

\footnotetext{
${ }^{1}$ Other acceptable terms are gathering power and angular acceptance
} 
- $d \vec{\sigma}$ is the infinitesimal element of area of the last sensor with total area $S$,

- $d \omega$ is the infinitesimal element of solid angle inside the domain $\Omega(\theta, \phi)$,

- $\vec{x}$ is the spatial coordinate of the telescope,

- $\hat{r}$ is the unit vector in the direction of $\omega$, and

- $\hat{r} \cdot d \vec{\sigma}$ is the effective element of area looking into $\omega$.

Although equation (2.1) is quite general, there are still some assumptions that must be taken into account, like

1. $d \vec{\sigma}, \omega$ and $\vec{x}$ are all time independent, because the telescope is at rest;

2. There are not any decays or transformation of particles through the passage by the detector, unless it is specified in $\varepsilon_{\alpha}$;

3. There is not any kind of process that could deviate the particle linear trajectory;

4. $J_{\alpha}$ is independent of $\sigma$ and $\varepsilon_{\alpha}$ is independent of $\vec{x}$.

To further simplifications lets consider an ideal telescope with its efficiency independent of $\omega, \sigma$ and $t$ leading to

$$
\begin{aligned}
& \varepsilon_{\alpha}=0, \quad \alpha \neq 1 \\
& \varepsilon_{1}=1, E_{i} \leq E \leq E_{f} \\
& \varepsilon_{1}=0, \quad \text { otherwise. }
\end{aligned}
$$

Henceforth, the subscript can be dropped. Assuming that the spectral intensity of the particle has no dependance in $\vec{x}$ and $t$, it can be rewriten as

$$
\vec{J}(E, \omega)=J_{0}(E) K(\omega)
$$

which leads to a simplification of equation (2.1)

$$
C=\left[\int_{\Omega} d \omega \int_{S} d \vec{\sigma} \cdot \hat{r} K(\omega)\right] \int_{E_{i}}^{E_{f}} d E J_{0}(E)
$$

The aperture $\Gamma$ is then defined as the term between the square brackets in equation (2.4)

$$
\begin{array}{r}
\Gamma \equiv \int_{\Omega} d \omega \int_{S} d \vec{\sigma} \cdot \hat{r} K(\omega) \\
=\int_{\Omega} d \omega K(\omega) D(\omega)
\end{array}
$$

where $D(\omega)$ defines the Directional Response Function

$$
D(\omega) \equiv \int_{S} d \vec{\sigma} \cdot \hat{r}
$$


For rectangular detectors which defines a axis-simetrical telescope, the counting rate for individual particles is given by

$$
\frac{d N}{d t}=\int_{\Omega} d \omega I(\omega) D(\omega)
$$

Assuming that the radiated particles have an angular distribution $I(\omega)=I_{0} K(\omega)=I_{0} \cos ^{n} \theta$, $\cos \theta=Z / r$ (figure 1), then equation (2.7) can be rewrited as

$$
\frac{d N}{d t}=I_{0} \int_{\Omega} \cos ^{n} \theta d \omega \int_{S} d \vec{\sigma} \cdot \hat{r}=I_{0} \Gamma_{n}
$$

where $\Gamma_{n}$ is the already defined aperture. Therefore, the counting rate is proportional to the telescope geometry with $I_{0}$ defining the vertical flux of particles passing through it.

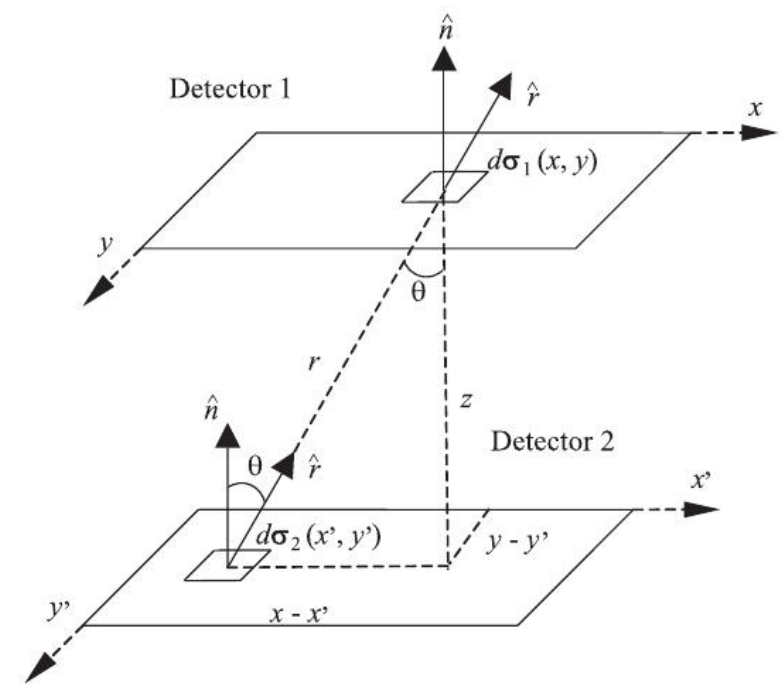

Figure 1: Schematic arrangement of detectors for the calculation of aperture. Extracted from [4].

The integration of $D(\omega)$ (equation(2.6)) for an element of area $d \sigma_{2}=d x \prime d y \prime$ (figure 1), returns the directional response in function of the detector's geometrical parameters $\mathrm{X}, \mathrm{Y}$ and $\mathrm{Z}$ :

$$
\begin{array}{r}
D(\omega)=\int_{S} d \vec{\sigma} \cdot \hat{r}=\int \cos \theta d x^{\prime} d y \prime \\
=\cos \theta(X-|Z \tan \theta \cos \phi|)(Y-|Z \tan \theta \sin \phi|)
\end{array}
$$

Applying equation (2.9) in (2.7), an expression for the counting rate in the $\omega$ direction is defined:

$$
\frac{d^{2} N}{d \omega d t}=I_{0}=\cos ^{n+1} \theta(X-|\xi|)(Y-|\eta|)
$$

where

$$
\begin{aligned}
& \xi \equiv Z \tan \theta \cos \phi,(-X \leq \xi \leq X) \\
& \eta \equiv Z \tan \theta \sin \phi,(-Y \leq \eta \leq Y)
\end{aligned}
$$


Therefore, using equation (2.7), a final expression for the aperture $\Gamma_{n}$ can be obtained

$$
\begin{aligned}
\Gamma_{n}= & \frac{1}{Z^{2}} \int_{-X}^{X} \int_{-Y}^{Y} \cos ^{n+4}(X-|\xi|)(Y-|\eta|) d \xi d \eta \\
& =\frac{4}{Z^{2}} \int_{0}^{X} \int_{0}^{Y} \cos ^{n+4}(X-\xi)(Y-\eta) d \xi d \eta \\
= & 4 Z^{n+2} \int_{0}^{X} \int_{0}^{Y} \frac{(X-\xi)(Y-\eta)}{\left[Z^{2}+\xi^{2}+\eta^{2}\right]^{(n+4) / 2}} d \xi d \eta .
\end{aligned}
$$

\section{Experimental methodology}

\subsection{Experimental setup}

The muon telescope consists of three detectors of plastic scintillator with light guides coupled to photomultipliers (PMTs). The dimensions of these paddles were $0,4 \times 0,4 \times 0,05 \mathrm{~m}^{3}$ to the telescope from Campinas and $1.44 \times 0.17 \times 0.02 \mathrm{~m}^{3}$ to the telescope located at Batavia. Each detector is connected to a high voltage power supply adjusted to its optimum value (the one that ensures maximum efficiency of the PMT). Particles crossing the scintillators generate light pulses converted to analogical electric signals, processed with NIM and CAMAC standard modules, for discrimination, time coincidence and counting.

The rate of single counting, double (considering the outermost detectors) and triple coincidences were recorded every ten minutes for a period of 4 hours, giving a set of measurements for each height of the telescope, ranging from 0.48 to 1.93 meters in the experiment realized in Brazil, and ranging from 0.186 to 2.034 meters in the one realized in the U.S. . The efficiency of the telescope, $\varepsilon_{T}$, was evaluated taking the product $\varepsilon_{T}=\varepsilon_{1} \times \varepsilon_{2} \times \varepsilon_{3}$, where each $\varepsilon_{n}$ correspond to the efficiency of the paddles 1,2 and 3, with $\varepsilon_{n}=N_{T} / N_{D}$ (between triple and double coincidences for each arrangement of the detectors with the paddle of interest placed in the middle). All frequency data were normalized considering the efficiency of the telescope. We have to mention that during data taken the atmospheric pressure was monitored, but no significant modulations of the telescope counting rate was observed in correlation with amplitude of pressure variations.

The same procedure described above was done putting a layer of $5 \mathrm{~cm}$ of lead, in the case of the experiment of Unicamp and a layer of $5 \mathrm{~cm}$ of steel in the experiment realized at Fermilab, just above the last detector of the telescope, to select particles with energy $E>100 \mathrm{MeV}$ and $E>75$ $\mathrm{MeV}$, respectively.

\subsection{Estimation of the parameter $n$}

Given the dimensions of a telescope (X, Y and Z), the aperture $\Gamma_{n}$ can be calculated with equation (2.12) for any value of $n$. If $\Gamma_{n}$ is obtained for different values of $Z$ (the distance between the outermost detectors) and the counting rate of particles is also measured, it is possible to obtain the muon intensity trough relation (2.8), with a linear fit from counting rate vs. aperture data.

The parameter $n$ can be estimated repeating this previous calculation for different values of $n$ and evaluating the reduced $\chi^{2}$, normalized by the number of degrees of fredom $\left(\chi^{2} / \mathrm{NDF}\right)$, for each value of $n$. Figure 2 shows results for 5000 random values of $n$, selected within a suitable range, and the calculated $\chi^{2} / N D F$ for the telescopes described in the previous section. The best value for 


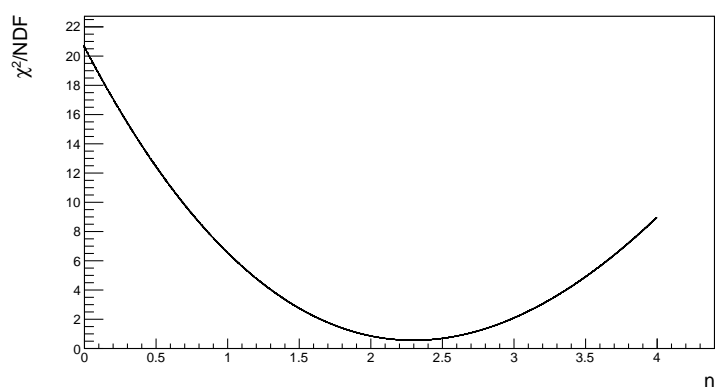

Figure 2: $\chi^{2} / N D F$ obtained for different values of $n$.

$n$ is chosen as the one which minimizes the $\chi^{2} / N D F$ and its deviation was defined by the $90 \%$ of confidence level region.

\section{Results}

Figure 3 shows the results obtained for both telescope assemblies, with no energy selection and with the lead absorber. The non-linear dependence of the counting frequency on telescope sensors separation is in agreement with the expected values from equations 2.8 and 2.12. It can also be seen that there is a global down-shift in data from the case we used the lead absorber, thus consistent with the energy cut applied.

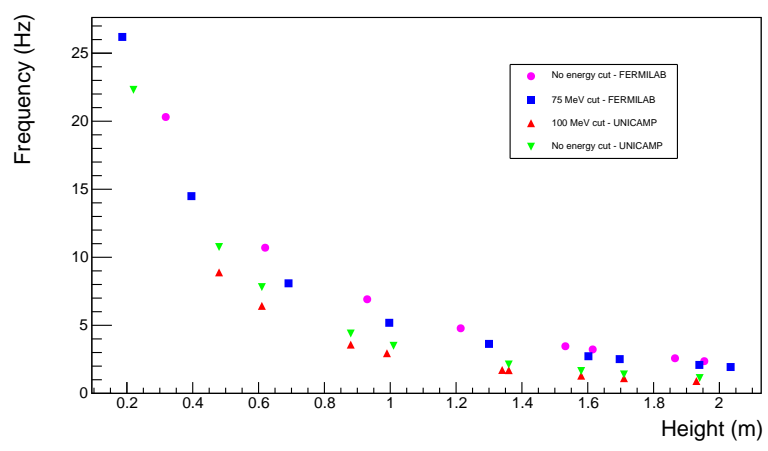

Figure 3: Data taken with the muon telescopes described in section 4.

Following the method described in section 3, the value of $n$ which minimizes the $\chi^{2} / N D F$ of the linear fit from frequency vs. aperture was calculated for data acquired with both assemblies for each experiment. These fits are shown in figures 4, 5, 6 and 7 for the optimum value of $n$. The results for $n$ and the intensity $I_{0}$ are summarised in table 1 . As mentioned before, deviations in $n$ were determined by the $90 \%$ of confidence level region whereas the flux deviations were obtained straight from the error for the angular coefficient of the linear fit.

The results obtained at Unicamp are consistent with other measurements [5] and also selfconsistent. But the results obtained at Fermilab are very different of the expected. These discrepancies may be due to the measurements on different seasons; the data take in Brazil was performed on January and February (that is Summer) and the data was taken on December and January on 


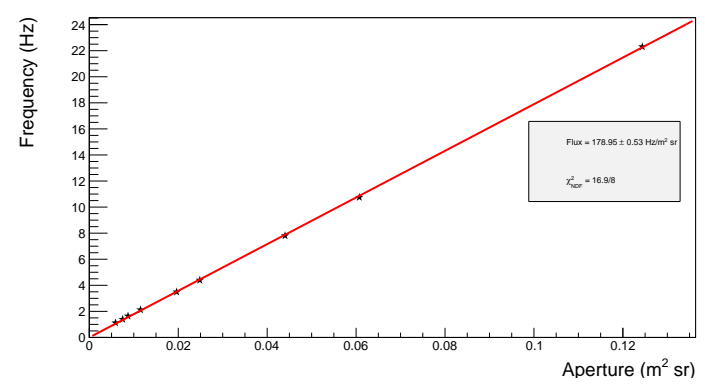

Figure 4: The best fit for the frequency dependence with the telescope at Unicamp without a lead absorber.

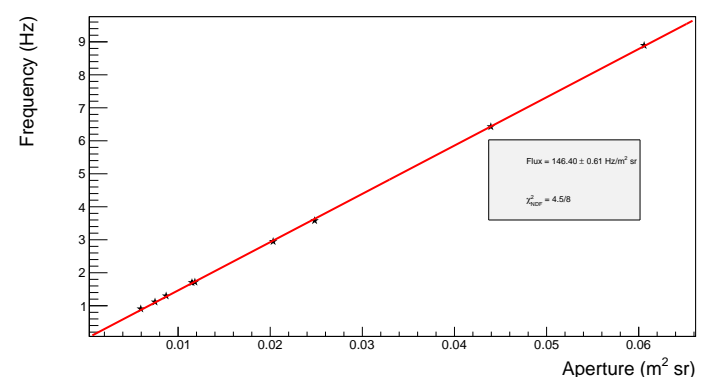

Figure 5: The best fit for the frequency dependence with the telescope at Unicamp with a lead absorber.

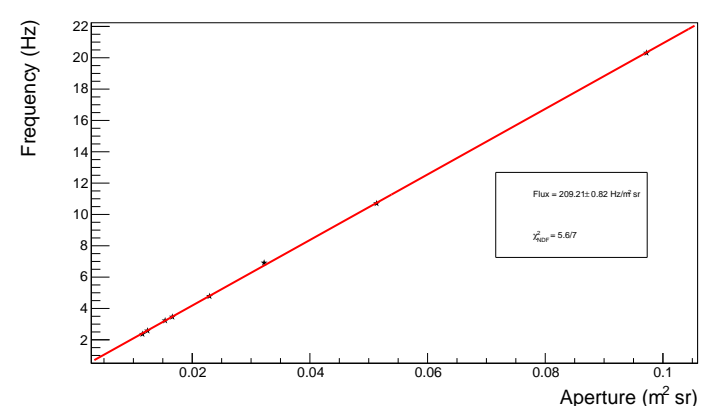

Figure 6: The best fit for the frequency dependence with the telescope at Fermilab without a steel absorber.

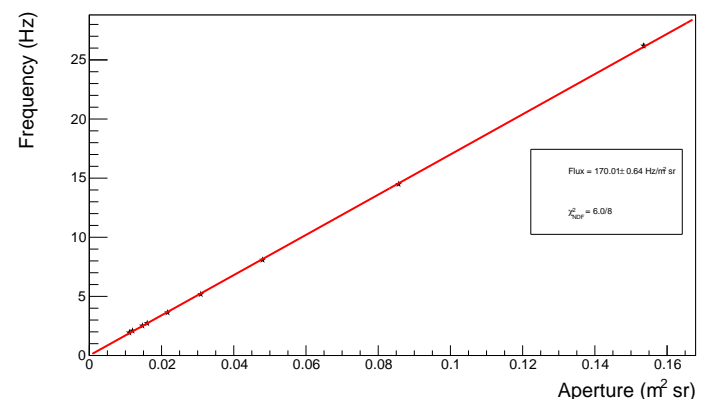

Figure 7: The best fit for the frequency dependence with the telescope at Fermilab with a steel absorber. 
Table 1: Values of $n$ and Intensity $\left(I_{0}\right)$ for each assembly of both experiments.

\begin{tabular}{cccc}
\hline Energy Cut & $n$ & $I_{0}\left(\mathrm{~Hz} / \mathrm{m}^{2} s r\right)$ & $\chi^{2} / N D F$ \\
\hline No cut (Unicamp) & $\left(2.27_{-0.12}^{+0.11}\right)$ & $(178.95 \pm 0.53)$ & $16.9 / 8$ \\
$100 \mathrm{MeV}$ & $\left(2.30_{-0.36}^{+0.36}\right)$ & $(146.40 \pm 0.61)$ & $4.5 / 8$ \\
No cut (Fermilab) & $\left(4.47_{-0.58}^{+0.63}\right)$ & $(194.87 \pm 0.76)$ & $5.6 / 7$ \\
$75 \mathrm{MeV}$ & $\left(3.66_{-0.36}^{+0.34}\right)$ & $(158.33 \pm 0.59)$ & $6.0 / 8$
\end{tabular}

Fermilab (that is winter). It's well know that the muon intensity has dependence with atmospheric conditions, like temperature and pressure [6].

\section{Summary}

Generally, the muon signals and backgrounds in particle physics experiments are estimated assuming the index $n$ of zenith angle dependence as $n=2$, but $n$ may depend on the location of the measurements and other conditions. A rather simple and fast method to determine local values for the $\cos ^{n}$ dependence of the muon flux in the surface was described. It can be done with a telescope with variable distance between the outermost detectors and not requires any further inclined detector arrangement. With this simple method, it is possible to simultaneously measure $n$ and the vertical flux, avoiding assumptions about generic and/or typical values.

Our measurements of the muon intensity in Campinas, Brazil and Batavia, U.S., with plastic scintillator detectors and with an absorber, result in the following values: $I_{0}=(146.40 \pm$ $0.61) \mathrm{Hz} / \mathrm{m}^{2} s r$, and $n=\left(2.30_{-0.36}^{+0.36}\right)$ for Campinas and $I_{0}=(158.33 \pm 0.59) \mathrm{Hz} / \mathrm{m}^{2} s r$, and $n=$ $\left(3.66_{-0.36}^{+0.34}\right)$, for Batavia. This experimental results are compatible with other measurements [5], and are different from the expected value for $n$, which is usually taken as $n=2$ as discussed in the text, and the discrepancy may be due to weather conditions [6].

These measurements can be easely performed in different locations, providing a broader range of physical measurements beyond the muon flux intrinsic features such as zenith angle dependence or vertical intensity. Possible observations in variations of these parameters can be related to height, latitude, pressure and energy cut-off effects induced by phenomena like the geomagnetic anomaly, injection of coronal mass, changes in the solar activity cycle and weather. More experiments are going to be performed to verify the dependence with the temperature and geographical locations. In a future work, a compilation of these results will be presented.

Acknowledgment: The colaborators thank CAPES and CPG for several financial supports.

\section{References}

[1] Pierre Auger Collaboration, The surface detector system of the Pierre Auger Observatory, Nuc. Inst. Meth. 586, 409-420, 2008.

[2] O. C. Allkofer, Introduction to Cosmic Radiation, University of Kiel, Germany, 1975.

[3] J.D. Sullivan, Geometric factor and directional response of single and multi-element particle telescopes, Nuc. Instr. Meth. 95, 5-11, 1971. 
[4] A.C. Fauth, J.C. Penereiro, E. Kemp, W.C. Grizolli, D.M. Consalter and L.F.G. Gonzales, Demonstra cão experimental da dilata cão do tempo e da contra ̃̃ ğão do espa ̃̃ ğo dos mÃžons da radia Ã $\breve{A} \tilde{c}$ c c Ãşsmica, Revista Brasileira de Ensino de Física 29, 4, 2007.

[5] M. Bektasoglu \& H. Arslan, Investigation of the zenith angle dependence of cosmic-ray muons at sea level, Pramana Journal of Physics 80, 5, 2013.

[6] Dmitrieva, A. N. et al. Temperature effect correction for muon flux at the earth surface: estimation of the accuracy of different methods. Journal of Physics: Conference Series, n. 409, 2013. 